W1E-(12)-4

\title{
SOFTWARE DISPERSION COMPENSATION IN OPTICAL COHERENCE TOMOGRAPHY
}

\author{
Chih-Wei Lu, Meng-Tsan Tsai, Yih-Ming Wang, Chia-Wei Sun, I-Jen Hsu, Yean-Woei Kiang \\ and C. C. Yang \\ Graduate Institute of Electro-Optical Engineering and Department of Electrical Engineering, \\ National Taiwan University, 1, Roosevelt Road, Section 4, Taipei, Taiwan \\ (phone) 886-2-23657624 (fax) 886-2-23652637 (E-mail) ccy@cc.ee.ntu.edu.tw
}

\begin{abstract}
We demonstrate a new method for dynamically and efficiently compensating the fundamental and high-order dispersion mismatches in an optical coherence tomography system based on multiple scans with split spectral bands and software process.
\end{abstract}

In this paper, we demonstrate a novel dispersion compensation method, in which OCT images are first obtained with segmented light source spectra. Then, an appropriate combination of these images can result in a high-quality image, in which dispersion is significantly compensated. In the OCT system, a super-luminescence diode is used as the light source with the center wavelength at $950 \mathrm{~nm}$ and the bandwidth of $70 \mathrm{~nm}$. The theoretical resolution is $5.6 \mu \mathrm{m}$. In the reference arm, an optical phase delay line is used for group- and phase-delay modulations. To match the optical paths between the reference and sample arms, an about $25 \mathrm{~cm}$ fiber length difference is required. In such a dispersive fiber, optical paths are different between different wavelengths within the $70 \mathrm{~nm}$ spectral width. In the proposed approach, we use a spatial filter, located between the lens and the galvanometer, for gating different wavelength bands of the light source spectrum. We conduct the longitudinal scan with each spectral band. Due to dispersion, the interference fringes of different spectral bands occur at different longitudinal positions. After properly adjusting the positions by software, the dispersion mismatch can be compensated. To improve the system sensitivity and imaging time, we reduce the distance between the lens and grating to $9 \mathrm{~cm}$. With this arrangement, the width of the interference fringe envelope is reduced to $31 \mu \mathrm{m}$ (see Fig. 1). With six split spectral bands, six interference fringe patterns from the six longitudinal scans of a glass surface with six successive split spectral bands $(20 \mathrm{~nm}$ in spectral width for each band) are obtained. The widths of those interference fringe envelopes are all about $20 \mu \mathrm{m}$, which is close to the theoretical limit. After properly shifting the positions of the six interference fringe patterns to align their envelope maximum positions. The summation of these six fringe patterns results in a new one of smaller envelope width, in which dispersion compensation has been significantly compensated. The result is shown in Fig. 2. The envelope width is now reduced to $7 \mu \mathrm{m}$. In the demonstration above, the A-mode scan is repeated for six times. Therefore, the costs we paid for software dispersion compensation include the longer imaging time and lower sensitivity. Nevertheless, the interference fringes of the split spectral bands can not only be used for compensating system dispersion, but also provide frequency-dependent dispersion information. Similar to the concept of spectral $\mathrm{OCr}$, absorption in a certain spectral window inside a sample can be obtained by comparing the intensities of the six images. Besides, if we plot the variation of the envelope maximum among the six fringes, we can obtain the wavelength-dependent group delay in the OCT system Furthermore, if we include the concept of spectral shaping, we can even reduce the fringe envelope width down to $5.6 \mu \mathrm{m}$, which is close to the theoretical limit.

Acknowledgement

This research was supported by the National Health Research Institute, The Republic of China, under the grant of NHRI-EX92-9220EI.

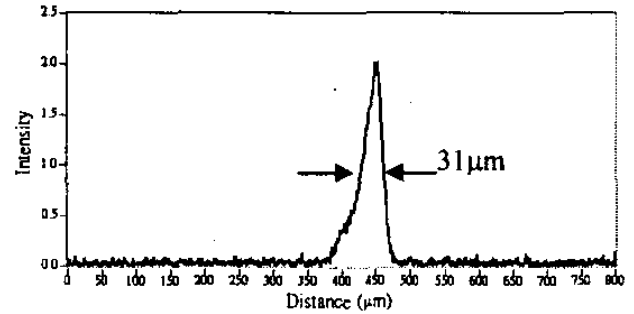

Fig. 1 Interference fringe envelope after decreasing the distance between the grating and lens in the optical phase delay line. The width is $31 \mu \mathrm{m}$.

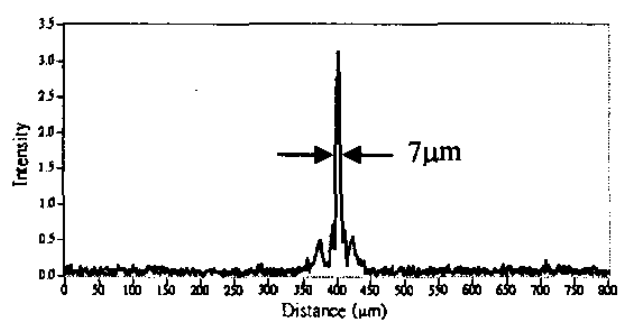

Fig. 2 Interference fringe envelope (reduced to $7 \mu \mathrm{m}$ ) after software process. 